\title{
Prévention du suicide
}

\section{Jean Martin}

Dr méd., membre de la rédaction

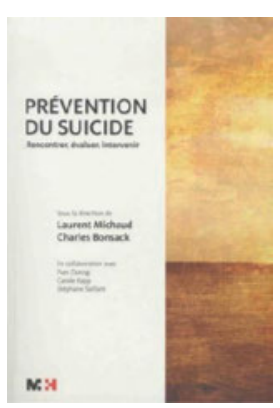

Sur un sujet de grande importance clinique et de santé publique, près de 80 auteurs ont contribué à ce livre fourni, précis. Moitié de femmes et moitié d'hommes, attachés à des services du CHUV pour deux tiers d'entre eux. La plupart sont psychiatres-psychothérapeutes. Une trentaine sont d'autres disciplines: notamment psychologues-psychothérapeutes, infirmiers, quelques médecins non-psychiatres.

Huit parties comprenant 41 chapitres. La première présente le contexte global du suicide, la seconde le modèle de rencontre, évaluation et intervention utilisé. On différencie trois niveaux: la prévention universelle (visant toute la population), la prévention sélective (à l'endroit de groupes à risque) et la prévention dite indiquée (pour ceux à risque manifeste). Sont évoqués l'intervention scolaire, les programmes sentinelles (gatekeepers) formant des bénévoles ou des professionnels, la coopéra-

\section{Sur un sujet de grande importance clinique et de santé publique, 80 auteurs ont contribué à cette somme riche et pratique.}

tion avec les médias, la prévention situationnelle (limitation de l'accès aux moyens - considération majeure de santé publique, qu'il s'agisse de médicaments, d'alcool ou d'endroits qui se prêtent au suicide - et bien sûr, si nous étions aux USA, il faudrait limiter drastiquement la disponibilité des armes à feu). Les auteurs insistent sur l'importance d'inscrire programmes et mesures dans une stratégie globale et dans la durée - ce qui implique d'anticiper les obstacles et les enjeux financiers et d'obtenir un soutien politique au meilleur niveau.

Les parties suivantes abordent chacune une problématique spécifique: Suicide et... périodes de la vie,... problèmes de santé,... dispositifs de soins. Puis on traite des possibles déterminants sociaux et politiques et de postvention - pour limiter la récidive ou la contagion: impact du suicide auprès de l'entourage, chez les proches, les professionnels, en milieu scolaire. Parmi les sujets moins «classiques»: suicide et... spiritualité, formation, incarcération, migration, LGBT, violence contre autrui, addiction (à des substances, aux jeux de hasard et d'argent); dans les troubles du comportement alimentaire; en périnatalité.

Chacun de ces chapitres (chacun «pièce d'un large puzzle») s'organise selon la même structure: 1) vignette clinique; 2) Que faut-il savoir? (point sur les connaissances); 3) Comment rencontrer et évaluer? (particularités de la situation clinique présentée); 4) Comment intervenir? (ce qui est efficace ou l'est moins); 5) A quoi être attentif? (notamment, erreurs à éviter); 6) le suivi/l'issue de l'histoire présentée.

Dans la préface (d'une Québécoise et d'un Lyonnais): «Dans chaque cas, le lecteur est confronté à une situation spécifique qui lui montre la prévention en mouvement, de l'impasse d'une souffrance insurmontable à l'ouverture d'un chemin qui préserve la vie.» «Il nous est aisé de faire partager notre enthousiasme. Ouvrage homogène et plein de vie, avec une colonne vertébrale robuste (...) Le lecteur est sensibilisé à la vulnérabilité suicidaire, telle qu'elle apparaît aux professionnels de la santé et du social (...) Les auteurs partagent le même référentiel d'évaluation, en distinguant le risque, l'urgence et la dangerosité suicidaires - langage commun de transversalité.» Le choix de procéder par entrées multiples permet à des acteurs différents d'accéder rapidement à des connaissances et recommandations pertinentes.

Une question - en toute humilité: dans un ouvrage sur la prévention, il est logique que les cas présentés se terminent habituellement bien, ou pour le moins permettent d'espérer une stabilisation. Aurait-il été pertinent/utile de discuter des situations où des efforts compétents d'aide et de prévention n'ont pas rencontré le succès? (cela est fait partiellement à propos de postvention).

A noter enfin que le dernier chapitre traite du suicide assisté. J’ai (J.M.) apprécié, en huit pages, une présentation basée sur l'expérience professionnelle et humaine de l'auteure, factuelle, non jugeante, équilibrée quant aux enjeux éthiques et pratiques que pose cette problématique - qu'il n'est plus possible aujourd'hui d'ignorer, ni de vouer aux gémonies. 